\title{
Thank you to our reviewers 2017
}

The editor would like to publicly acknowledge the people listed below who served as reviewers on the journal during 2017. Without their efforts, the quality of the journal could not be sustained.

$\begin{array}{lll}\text { Akil, Hussein } & \text { Burnett, John } & \text { Dao, Doan } \\ \text { Auchus, Richard } & \text { Calatayud, Dan } & \text { Das, Samrat } \\ \text { Baer, stephanie } & \text { Caulfield, Michael } & \text { Davis, Dawn } \\ \text { Bajaj, Jas } & \text { Celik, Gulperi } & \text { De Lima, Thais Martins } \\ \text { Barnes, John } & \text { Chang, Alice } & \text { Devlin, James } \\ \text { Belo, Vanessa } & \text { Chayanupatkul, Maneerat } & \text { DiMaio, Michael } \\ \text { Berg, Gabriela } & \text { Chen, Yu } & \text { Dutcher, Janice } \\ \text { Bottillo, Irene } & \text { Colburn, Keith } & \text { Elisei, Rossella } \\ \text { Boufraqech, Myriem } & \text { Concato, John } & \text { Franklin, Oskar } \\ \text { Burge, Mark } & \text { Crook, Errol } & \text { Garshick, Eric } \\ \text { Burgess, Tom } & \text { Cunningham, Glenn } & \text { Ghayee, Hans }\end{array}$

Giulietti, Matteo

Gosmanov, Aidar Grant, Struan Grau, Veronika

Gregg, Lucile Gupta, Samir Gurka, Matthew Haley, Robert 